\title{
Applying Choosing Wisely: Antinuclear Antibody (ANA) and Sub- Serology Testing in a Safety Net Hospital System
}

\author{
Lisa Anne Davis ${ }^{*}, 1,2$, Barbara Goldstein ${ }^{1,2,3}$, Vivian Tran ${ }^{1,2}$, Angela Keniston ${ }^{1}$, Jinoos Yazdany ${ }^{4}$, Joel \\ Hirsh $^{1,2}$, Amy Storfa ${ }^{1}$ and JoAnn Zell ${ }^{1,2,3}$
}

${ }^{I}$ Denver Health and Hospital Authority, Denver, CO, USA

${ }^{2}$ University of Colorado School of Medicine, Aurora, CO, USA

${ }^{3}$ National Jewish Health, Denver, CO, USA

${ }^{4}$ University of California San Francisco, San Francisco, CA, USA

\begin{abstract}
Objective: In 2013, the American College of Rheumatology (ACR) participated in the Choosing Wisely campaign and devised a recommendation to avoid testing antinuclear antibody (ANA) subserologies without a positive ANA and clinical suspicion of disease. The goals of our study were to describe ANA and subserology ordering practices and predictors of ordering concurrent ANA and subserologies in a safety-net hospital.

Methods: We identified ANA and subserologies (dsDNA, Sm, RNP, SSA, SSB, Scl-70 and centromere) completed at Denver Health between $1 / 1 / 2005$ and 12/31/2011. Variables included demographics, primary insurance, service, and setting from which the test was ordered. We performed multivariable logistic regression to determine predictors of concurrent ordering of ANA and subserologies.

Results: During seven years, 3221 ANA were performed in 2771 individuals and $211(6.6 \%)$ were performed concurrently with at least one subserology. The most common concurrent subserologies were dsDNA (21.8\%), SSA (20.8\%), and SSB (19.7\%). In the multivariable logistic analysis, significant predictors of concurrent ANA and subserologies were the labs being ordered from subspecialty care (OR 8.12, 95\% CI 5.27-12.50, p-value $<0.0001$ ) or from urgent/inpatient care (OR 3.86, 95\% CI 1.78-8.38, p-value 0.001). A significant predictor of decreased odds was male gender (OR 0.32, 95\% CI $0.21-0.49$, p-value $<0.0001)$. Five individuals $(2.2 \%$ of the negative ANA with subserologies ordered) had a negative ANA but positive subserologies.

Conclusion: Of 3221 ANA, 6.6\% were performed concurrently with subserologies, and subspecialists were more likely to order concurrent tests. A negative ANA predicted negative subserologies with rare exceptions, which validates the ACR's recommendations.
\end{abstract}

Keywords: Antinuclear antibody (ANA), choosing wisely, ordering practices, quality of care, quality indicators, resource management.

\section{INTRODUCTION}

In 2013, Yazdany et al. [1] published the Choosing Wisely Top 5 recommendations for the American College of Rheumatology (ACR). These recommendations were in response to the American Board of Internal Medicine Foundation's Choosing Wisely campaign, which encouraged medical professional societies to propose a list of five tests, treatments, or services that are commonly used, but the usefulness of which should be reevaluated by clinicians and patients. One of the ACR's Top 5 recommendations was "Do not test antinuclear antibody (ANA) subserologies without a positive ANA and clinical suspicion of immunemediated disease" [1]. This recommendation was made in the setting of previous work, which indicated that with the

*Address correspondence to this author at the Denver Health and Hospital Authority, 777 Bannock St. MC 4000, Denver, Colorado 80204-4507, USA; Tel: (303) 602-5037; Fax: (303) 602-5055; E-mail: lisa.davis@ucdenver.edu use of the HEp-2 cell line, ANA tests are very sensitive (9399\%) [2-4] for the diagnosis of systemic lupus erythematosus (SLE). Historically, ANA tests using non-human substrates can lose the ability to detect SSA, thus producing false negative results [5-8]. However, the human-derived HEp-2 cell line, which can be transfected with the SSA antigen [9], is thought to be more sensitive than non-human substrates, although not all laboratories utilize transfected cells.

In 2000, guidelines issued by the American College of Rheumatology along with members of the College of American Pathologists stated "using HEp-2 cells as a substrate has virtually eliminated false-negative ANA results. Aside from rare cases, further autoantibody testing, if a patient has a negative ANA finding, is not indicated" [10]. Similarly, in 2002, the Italian Society of Laboratory Medicine [11] developed guidelines for laboratory testing and stated that "in the case of a negative ANA... [further] antinuclear specific antibody [subserology] testing is only indicated when a patient has clear signs of an autoimmune 
rheumatic disease". Thus, the ANA item in the ACR's Top 5 list asserts that: "Tests for ANA subserologies (including antibodies to double-stranded DNA, Sm, RNP, SSA, SSB, Scl-70, and centromere) are usually negative if the ANA is negative. Exceptions include anti-Jo-1, which can be positive in some forms of myositis, or occasionally, anti-SSA in the setting of lupus or Sjogren's syndrome. Broad testing of autoantibodies should be avoided; instead, the choice of autoantibodies should be guided by the specific disease under consideration" [1].

These recommendations have been supported by some small-scale studies. In one study, ANA subserologies were reviewed in 153 individuals who were found to have a negative ANA screen, and no positive subserologies were detected [12]. In another study, ANA subserologies (SSA, $\mathrm{SSB}, \mathrm{Sm}, \mathrm{RNP}$ ) were examined in 468 patients with a negative ANA. Three patients were identified who were consistently ANA negative, but had at least one positive subserology $(0.64 \%)$ [13].

Complicating these recommendations, however, is each individual laboratory's choice of ANA testing technique. Frequently, laboratories choose enzyme-linked immunosorbent assays (ELISA) as an initial screen, which is then verified by indirect fluorescent assay (IFA) using the HEp-2 cells. This practice is often utilized clinically due to the labor-intensive nature of the IFA. One recent publication showed that one particular laboratory's ELISA had a negative predictive value (NPV) of 0.92 in SLE [14]. While a NPV of 0.92 is quite good, it does leave room for some individuals who might have a negative ELISA ANA but a positive IFA ANA.

Recently, some laboratories have offered an "arthritis" panel, which provides an opportunity to test a number of subserologies at once, including ANA and ANA subserologies. Alternatively, some laboratories offer ANA reflex testing, which cancels the ANA subserologies if the ANA is negative. While ANA reflex testing likely saves money compared to arthritis panels, not all laboratories offer reflex panels, and the reflex panels are not symptom-guided. This Top 5 item specifically argues against the use of such panels as they may be used for non-specific symptoms and may result in unnecessary testing and false positive tests. To our knowledge, ordering patterns of ANA and subserologies have not been recently investigated.

To address this gap in knowledge, we examined the ordering patterns for ANA and ANA subserologies in Denver Health, a large safety-net hospital in Denver, Colorado. Denver Health is in an interesting position to examine this recommendation, as each subserology must be ordered individually, as opposed to a reflex or arthritis panel. We felt that this particular institutional requirement offered a unique window into the practice patterns of general and subspecialty clinicians. Our specific goals were to: 1) evaluate the ordering practices of our health system, focusing on concurrent ANA and subserology orders; 2) investigate predictors of concurrent ordering of ANA and subserologies; and 3) report the outcomes in which a patient was found to be ANA negative but have a positive subserology.

\section{MATERIALS AND METHODS}

\section{Study Population and Variables}

We identified all ANA and subserologies (dsDNA, Sm, RNP, SSA, SSB, Scl-70, and centromere) that were completed at Denver Health between January 1, 2005 and December 31, 2011 via query of the electronic medical record. We identified additional demographic variables, including: age at the time of antibody testing; gender; the setting from which the test was ordered (outpatient, lab draw alone, emergency or urgent care, or inpatient); the service that ordered the test (primary care, specialty service, or other [emergency/urgent care or inpatient care]); race/ethnicity (Hispanic, Caucasian, African American, other/unknown); and primary payer (medically indigent, Medicaid, Medicare, commercial insurance, self-pay, or other/unknown).

ANA ordered at Denver Health are sent to a national reference laboratory (Quest or ARUP) where specimens are screened using an enzyme-linked immunosorbent assay (ELISA) method, detecting IgG-specific antibodies. ELISA screen results that are reported as "detected," based on optical density, are followed by indirect fluorescent assay (IFA) using HEp-2 cells with an IgG-specific conjugate. Reflexive IFA testing is reported as a titer with a reference range of $<1: 40$. ANA subserologies are performed using multiplex bead technology with reference intervals established by the manufacturer.

We performed chart reviews for the individuals with consistently negative ANA, but at least one positive subserology.

\section{Statistical Analysis}

We used descriptive statistics to analyze patterns of ANA testing, and utilized logistic regression to determine factors associated with concurrent ordering of ANA and at least one subserology test. We first examined variables as univariate analyses, and we included those variables in the multivariable analysis if the p-value was $\leq 0.2$ in the univariate model. We considered variables to be significant in the multivariable model if the p-value was $\leq 0.05$. All analyses were performed using STATA version 13.1 (College Station, TX).

\section{Ethics}

This project was submitted to the Colorado Multiple Institutional Review Board and was considered not human subject research, but rather a quality improvement project.

\section{RESULTS}

During the seven-year time period, 3221 ANA were performed in 2771 individuals. In the majority of the individuals, (2400 or $86.6 \%$ ), only one ANA was ordered. Two ANA were ordered in 305 individuals (11.0\%), while 3 were ordered in 55 individuals $(2.0 \%), 4$ in 9 individuals $(0.3 \%)$, and 5 in 2 individuals $(0.1 \%)$.

Of these 3221 ANA performed, $211(6.6 \%)$ were done concurrently with at least one subserology (see Fig. 1). The most common concurrently ordered subserologies with an 


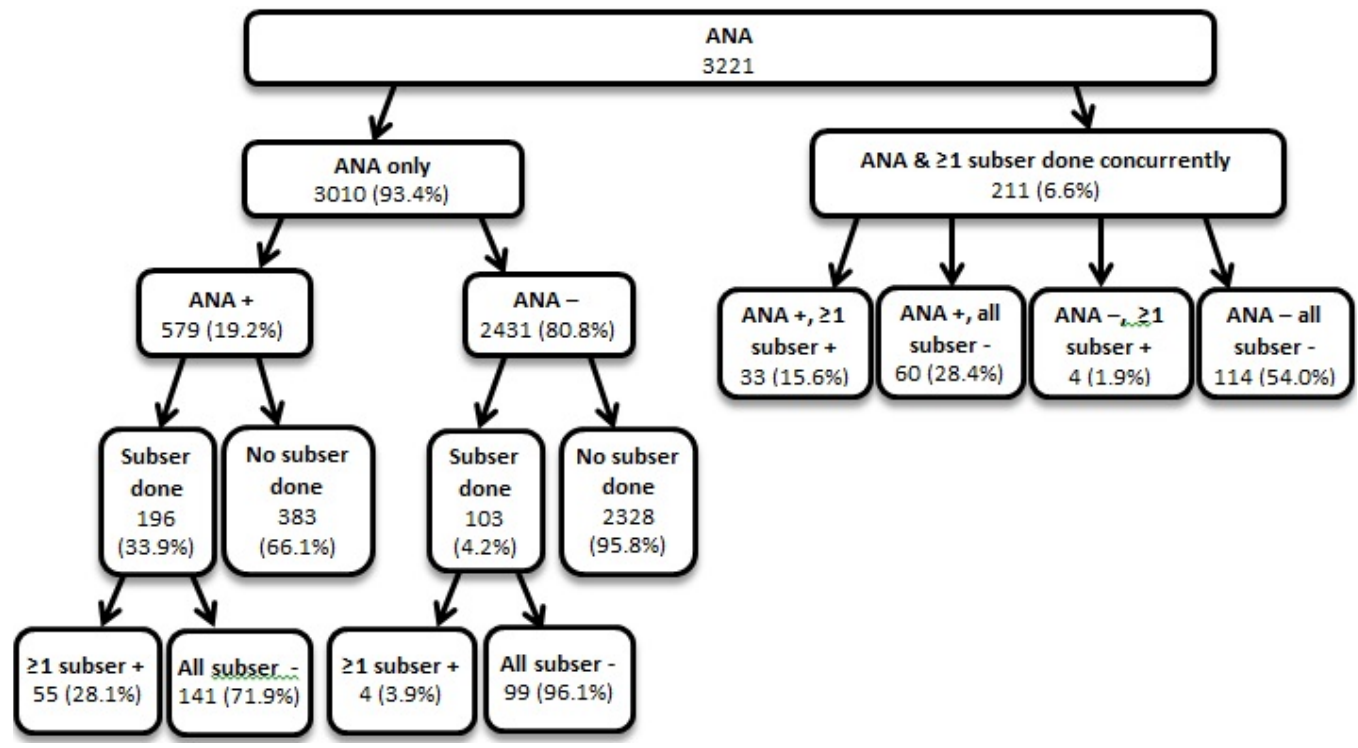

ANA: antinuclear antibody; Subser: subserology antibodies include SSA (Ro), SSB (La), Scl-70, dsDNA, Smith, centromere, and ribonucleoprotein (RNP). +: positive, as defined by the manufacturer; -: negative, as defined by the manufacturer

Fig. (1). ANA and subserologies, with order of events.

ANA were dsDNA (21.8\%), SSA (20.8\%), SSB (19.7\%), Sm (14.08\%), RNP (13.03\%), Scl-70 (7.22\%), and

centromere $(3.35 \%)$. Of the 3010 ANA that were performed initially without subserologies, $579(19.2 \%)$ were positive and $2431(80.8 \%)$ were negative. Of the positive ANA that were performed without subserologies, 383 (66.1\%) did not have subsequent subserologies performed, while 196 $(33.9 \%)$ did. Of the 2431 with a negative ANA and without concurrent subserologies ordered, 103 (4.2\%) later had subserologies ordered.

The demographics by ANA performed are shown in Table 1. The mean patient age was 45.2 years (SD 14.4 years). The majority of the ANA were performed in Hispanics $(46 \%)$, and $89 \%$ of the ANA were performed in the outpatient setting. The majority of the ANA were ordered from the primary care service $(59 \%)$, and the most common primary payer source was the medically indigent program (47\%).

The results of the univariate and multivariable logistic regression models of predictors of concurrent ordering of an ANA and at least one subserology may be found in Table 2. In the multivariable logistic analysis, significant predictors of increased odds of ANA and subserologies being ordered concurrently were that the labs were ordered from subspecialty care (OR 8.12, 95\% CI 5.27-12.50, p-value $<0.0001$ ) or from other care (OR 3.86, 95\% CI 1.78-8.38, pvalue 0.001). A significant predictor of decreased odds of ANA and subserologies being ordered concurrently was male gender (OR 0.32, 95\% CI 0.21-0.49, p-value <0.0001).

In total, there were five individuals $(2.2 \%$ of the negative ANA with subserologies ordered) who had consistently negative ANA and positive subserologies. All five individuals were females, and ranging in age from 19-64 years. Subserologies found to be positive in these individuals include SSA (2), Scl-70 (2), RNP (1), and dsDNA (1). Four out of the five were diagnosed with a mild form of SLE or other connective tissue disease, while one individual was felt to have a possible paraneoplastic process in the setting of concomitant non-small cell lung cancer. The details of these cases may be found in Appendix Table A1.

Table 1. Demographics, by ANA ordered.

\begin{tabular}{|c|c|c|c|}
\hline Variable & $\mathbf{n}=$ & Mean, \% & SD \\
\hline Age, years (mean) & 3,221 & 45.2 & 14.4 \\
\hline Gender, male (\%) & 3,221 & 31.9 & \\
\hline \multicolumn{4}{|l|}{ Race/Ethnicity (\%) } \\
\hline Hispanic & 1,481 & 46.0 & \\
\hline Caucasian & 872 & 27.1 & \\
\hline African American & 677 & 21.0 & \\
\hline Other/Unknown & 191 & 5.9 & \\
\hline \multicolumn{4}{|c|}{ Setting in which ANA ordered (\%) } \\
\hline Outpatient & 2,381 & 89.4 & \\
\hline Lab draw alone & 226 & 8.5 & \\
\hline Emergency & 41 & 1.5 & \\
\hline Inpatient & 14 & 0.5 & \\
\hline \multicolumn{4}{|l|}{ Primary Payment Source (\%) } \\
\hline Medically Indigent & 1,354 & 46.5 & \\
\hline Medicaid & 741 & 25.4 & \\
\hline Medicare & 402 & 13.8 & \\
\hline Commercial Insurance & 336 & 11.5 & \\
\hline Self-pay & 48 & 1.7 & \\
\hline Other/Unknown & 33 & 1.1 & \\
\hline \multicolumn{4}{|l|}{ Service ordering ANA (\%) } \\
\hline Primary care & 1,704 & 58.5 & \\
\hline Subspecialty care & 1,010 & 34.7 & \\
\hline
\end{tabular}


Table 2. Univariate and multivariable logistic regression models of predictors of concurrent ordering of ANA and at least one subserology.

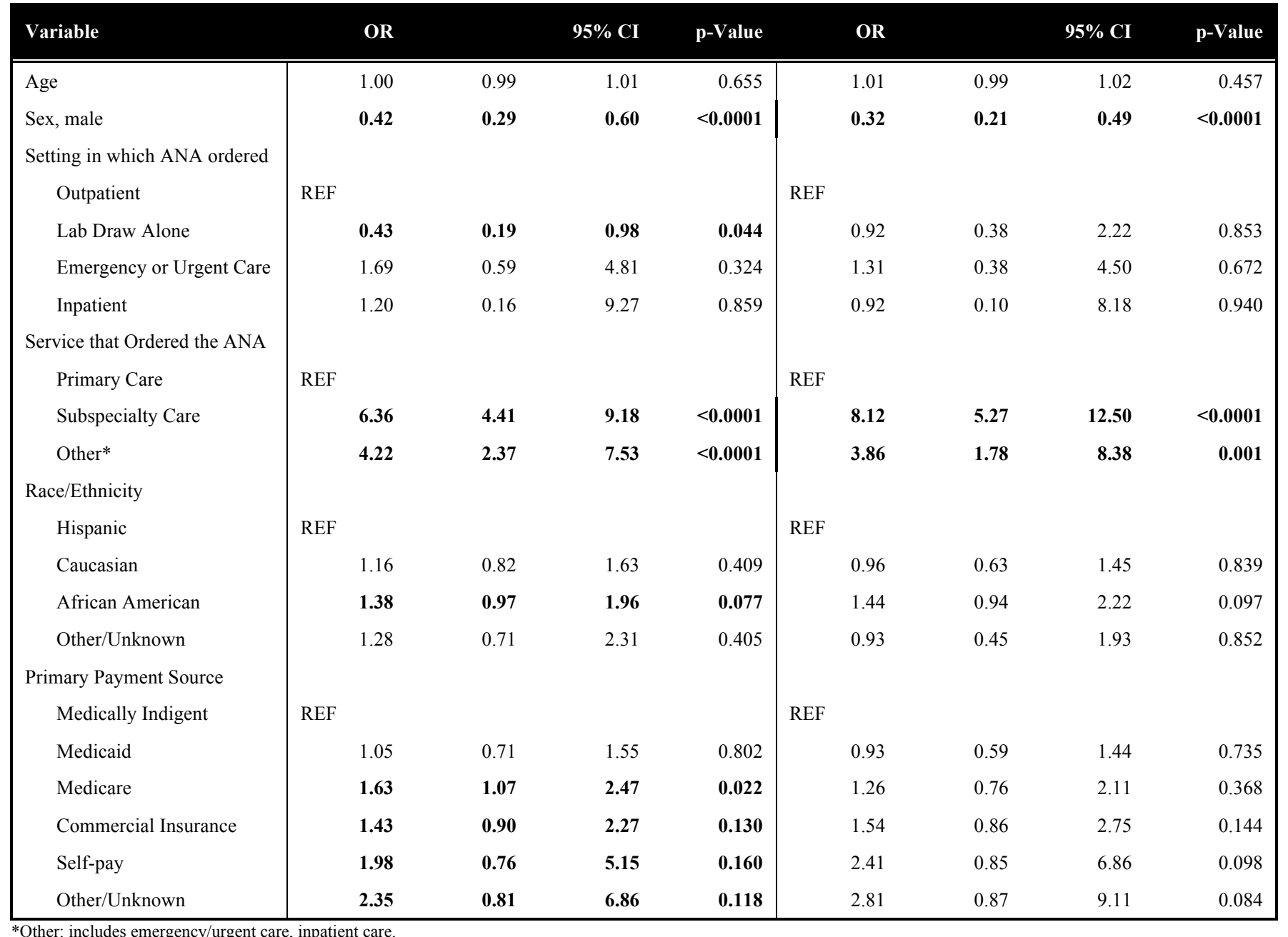

Other*

*Other: includes emergency/urgent care, inpatient care.

Variable included in the multivariable model if p-value $<=0.2$ in the univariate model; variable considered significant in the multivariable model if the $p$-value $<=0.05$.

\section{DISCUSSION}

We found that when providers are given a choice (i.e. they must order laboratory examinations individually), only $6.6 \%$ of ANA were ordered concurrently with a subserology. This indicates that providers at our institution typically do not overuse ANA subserology testing, but wait for the ANA to return before initiating subserology testing. This ordering pattern may be due to our hospital setting - a safety net hospital with a systematic effort toward efficient use of resources. The Top 5 recommendations acknowledge that clinical context dictates ordering patterns, and there likely are situations that warrant testing an ANA and subserologies at the same visit. These include but are not limited to: patient financial constraints with multiple co-pays; long travel times; and unclear reports of prior testing. The most likely subserologies to be ordered concurrently with an ANA were dsDNA, SSA, and SSB. While ordering SSA and possibly the SSB reflect possible pitfalls in ANA specificity [5-8], concurrent ordering of dsDNA does not. We propose this as an area for better education in our institution and a possible area of caution for other institutions.

Predictors of concurrent ordering of ANA and subserologies include that the labs were ordered from subspecialty care $(\mathrm{OR} 8.12$, p-value $<0.0001)$ or from other care, such as emergency/urgent care or inpatient care (OR 3.86 , p-value 0.001). We hypothesize that the increased concurrent ordering in subspecialty and other care may be driven by a high index of clinical suspicion for autoimmune disease and/or the long potential wait time to be seen in subspecialty clinics. That is, the clinicians in these clinics may want to maximize the laboratory information available when the patient is seen. While this approach may not be the most cost effective, it may be time-effective and decrease the time burden for patients.

A significant predictor of decreased odds of ANA and subserologies being ordered concurrently was male gender (OR 0.32, p-value $<0.0001$ ). Again, we suspect that this may be a reflection of clinicians' clinical suspicion, as the majority of immune-mediated diseases affect females [15].

Additionally, we found that only rarely were subserologies positive, when tested, in the setting of consistently negative 
ANA. We found 5 cases $(2.2 \%$ of negative ANA with subserologies ordered) in which an individual had a consistently negative ANA and at least one positive subserology. Of these cases, SSA and Scl-70 were the most likely subserologies to return as positive. The discrepancy between the negative ANA and positive serologies may be explained by the screening technique utilized by our clinical laboratory - the initial screen performed by ELISA and confirmed by IFA. Previous studies have indicated that screening with ELISA, while much cheaper and quicker, may not detect all positive ANAs found by IFA [14]. The Yazdany et al. paper [1] acknowledges the possibility of a positive SSA in the setting of a negative ANA, but Scl-70 positivity in the setting of a negative ANA is something that should be further explored. Additionally, we cannot speak to the subserologies of those individuals who had a negative ANA and no further testing was done. An important limitation of our paper is that the initial ANA screen is performed by ELISA and then confirmed by IFA. As discussed above, this excludes those individuals who have a negative ANA by ELISA but may have a positive ANA by IFA [14]. Other limitations of our study include the retrospective chart review nature, which is subject to channeling bias - i.e. only those individuals that the clinician had a suspicion of autoimmune disease were tested for ANA or further tested for subserologies. Therefore, we are unable to comment on the rates of positive or negative ANA and subserologies of the general population. Additionally, as is seen in retrospective studies, our study was based on clinically available data, which are subject to the clinical changes of data quality that occur over time.

Strengths of our study include the seven years of data accumulated, the large number of ANA performed, and the setting of the study in a safety-net hospital setting where cost is a daily concern. Additionally, our study reflects real-life clinical practice.

\section{CONCLUSION}

In summary, we feel that the ACR's Top 5 recommendation to not test ANA subserologies without a positive ANA and clinical suspicion of immune-mediated disease to be a valid recommendation. Only rarely did we find a patient who had a negative ANA and a positive subserology. In examining the ordering patterns of our clinicians at Denver Health, we did not see frank misuse of ANA testing. We did find that subspecialists and those involved in care in emergency/urgent/inpatient settings were more likely to concurrently order ANA and subserologies, however, clinical circumstances may have necessitated this approach in some cases. Future studies should examine other clinical settings where arthritis panels and/or ANA reflex testing are offered, or in settings where resources are not as closely guarded. Additional future studies also include a cost-analysis of the individual laboratory approach and sensitivity and specificity of ELISA $v s$ IFA for predicting ANA subserologies in our population. Examining variation in utilization across health systems with different payment models is another important next step.

\section{LIST OF ABBREVIATIONS}

$\begin{array}{ll}\text { ACR } & =\text { American college of rheumatology } \\ \text { ANA } & =\text { Antinuclear antibody } \\ \text { ELISA } & =\text { Enzyme-linked immunosorbent assay } \\ \text { IFA } & =\text { Indirect fluorescent assay } \\ \text { SLE } & =\text { Systemic lupus erythematosus }\end{array}$

\section{CONFLICT OF INTEREST}

The authors confirm that this article content has no conflict of interest.

\section{ACKNOWLEDGEMENTS}

Funding for this project was provided by: the American College of Rheumatology (ACR) Rheumatology Research Foundation (RRF) Rheumatology Scientist Development Award. Dr. Davis is supported by the ACR RRF Rheumatology Scientist Development Award, Denver Health and Hospital Authority, and the University of Colorado. Dr. Yazdany is supported by NIH/NIAMS K23 AR060259 and P60 AR053308 and Rosalind Russell Medical Research Center for Arthritis.

\section{APPENDIX}

Table A1. Consistently negative ANA, positive subserologies case details.

\begin{tabular}{|c|c|c|c|c|c|c|c|}
\hline Age & Gender & $\#$ Neg ANA & Pos Subserologies & Neg Subserologies & Manifestations & Diagnosis & Outcome \\
\hline 34 & $\mathrm{~F}$ & 2 & SSA, RNP & dsDNA, Sm, SSB & $\begin{array}{l}\text { leukopenia, anemia, } \\
\text { Raynaud's, arthralgias }\end{array}$ & SLE, FM & placed on $\mathrm{HCQ}$ \\
\hline 64 & $\mathrm{~F}$ & 1 & Scl-70 & cent, Jo-1 & $\begin{array}{c}\text { Raynaud's, } \\
\text { arthralgias, NSIP }\end{array}$ & $\begin{array}{l}\text { concomitant stage III } \\
\text { NSCLC; possible } \\
\text { paraneoplastic process }\end{array}$ & $\begin{array}{c}\text { died due to ARDS and } \\
\text { sepsis }\end{array}$ \\
\hline 19 & $\mathrm{~F}$ & 2 & SSA & & arthralgias, fatigue & SLE, FM & placed on $\mathrm{HCQ}$ \\
\hline 38 & $\mathrm{~F}$ & 6 & dsDNA & cent & $\begin{array}{c}\text { Raynaud's, alopecia, } \\
\text { livedo }\end{array}$ & possible SLE & $\begin{array}{c}\text { placed on HCQ, lost } \\
\text { to follow up }\end{array}$ \\
\hline 31 & $\mathrm{~F}$ & 2 & Scl-70 & $\mathrm{SSA}, \mathrm{SSB}, \mathrm{Sm}$ & Raynaud's & Raynaud's & $\begin{array}{l}\text { trial of HCQ not } \\
\text { effective }\end{array}$ \\
\hline
\end{tabular}

ANA: antinuclear antibody; Pos: positive; Neg: negative; dsDNA: double stranded DNA; cent: centromere; SLE: systemic lupus erythematosus; FM: fibromyalgia; HCQ: hydroxychloroquine; NSIP: non-specific interstitial pneumonia; NSCLC: non-small cell lung cancer; ARDS: acute respiratory distress syndrome. 


\section{REFERENCES}

[1] Yazdany J, Schmajuk G, Robbins M, et al. Choosing wisely: the American College of Rheumatology's Top 5 list of things physicians and patients should question. Arthritis Care Res (Hoboken) 2013; 65: 329-39.

[2] Gronhagen CM, Gunnarsson I, Svenungsson E, Nyberg F. Cutaneous manifestations and serological findings in 260 patients with systemic lupus erythematosus. Lupus 2010; 19: 1187-94.

[3] Kumar Y, Bhatia A, Minz RW. Antinuclear antibodies and their detection methods in diagnosis of connective tissue diseases: a journey revisited. Diagn Pathol 2009; 4: 1.

[4] Tanaka N, Muro Y, Sugiura K, Tomita Y. Anti-SS-A/Ro antibody determination by indirect immunofluorescence and comparison of different methods of anti-nuclear antibody screening: evaluation of the utility of HEp-2 cells transfected with the $60 \mathrm{kDa}$ SS-A/Ro as a substrate. Mod Rheumatol 2008; 18: 585-92.

[5] Ahmed AR, Workman S. ANA-negative systemic lupus erythematosus. Clin Exp Dermatol 1983; 8: 369-77.

[6] Kulick KB, Mogavero H, Jr., Provost TT, Reichlin M. Serologic studies in patients with lupus erythematosus and psoriasis. J Am Acad Dermatol 1983; 8: 631-4.

[7] Provost TT, Razzaque A, Maddison PJ, Reichlin M. Antibodies to cytoplasmic antigens in lupus erythematosus. Serologic marker for systemic disease. Arthritis Rheum 1977; 20: 1457-63.
[8] Ulvestad E, Kanestrom A, Madland TM, Thomassen E, Haga HJ, Vollset SE. Evaluation of diagnostic tests for antinuclear antibodies in rheumatological practice. Scand J Immunol 2000; 52: 309-15.

[9] Keech CL, Howarth S, Coates T, Rischmueller M, McCluskey J, Gordon TP. Rapid and sensitive detection of anti-Ro (SS-A) antibodies by indirect immunofluorescence of $60 \mathrm{kDa}$ Ro HEp-2 transfectants. Pathology 1996; 28: 54-7.

[10] Kavanaugh A, Tomar R, Reveille J, Solomon DH, Homburger HA. Guidelines for clinical use of the antinuclear antibody test and tests for specific autoantibodies to nuclear antigens. American College of Pathologists. Arch Pathol Lab Med 2000; 124: 71-81.

[11] Tozzoli R, Bizzaro N, Tonutti E, et al. Guidelines for the laboratory use of autoantibody tests in the diagnosis and monitoring of autoimmune rheumatic diseases. Am J Clin Pathol 2002; 117: 316-24.

[12] Slater CA, Davis RB, Shmerling RH. Antinuclear antibody testing. A study of clinical utility. Arch Int Med 1996; 156: 1421-5.

[13] Thomson KF, Murphy A, Goodfield MJ, Misbah SA. Is it useful to test for antibodies to extractable nuclear antigens in the presence of a negative antinuclear antibody on Hep-2 cells? J Clin Pathol 2001; 54: 413.

[14] Hira-Kazal R, Shea-Simonds P, Peacock JL, Maher J. How should a district general hospital immunology service screen for antinuclear antibodies? An 'in-the-field' audit. Clin Exp Immunol 2015; 180: 52-7.

[15] West S. Rheumatology secrets. $2^{\text {nd }}$ ed. Philadelphia: Hanley \& Belfus 2002 .

(C) Davis et al.; Licensee Bentham Open.

This is an open access article licensed under the terms of the Creative Commons Attribution Non-Commercial License (http://creativecommons.org/licenses/by-nc/ 4.0/) which permits unrestricted, non-commercial use, distribution and reproduction in any medium, provided the work is properly cited. 